EPJ Web of Conferences 38, 02002 (2012)

DOI: $10.1051 /$ epjconf/20123802002

(c) Owned by the authors, published by EDP Sciences, 2012

\title{
Damping of high-energy particle-hole-type nuclear excitations: a semi-microscopic model
}

\author{
M. H. Urin ${ }^{\mathrm{a}}$ \\ National Research Nuclear University “MEPhI”, 115409 Moscow, Russia
}

\begin{abstract}
A semi-microscopic model (particle-hole dispersive optical model) is formulated to describe the main relaxation modes of high-energy particle-hole-type excitations in medium-heavy mass nuclei. Within this model Landau damping and the single-particle continuum are considered microscopically, while the spreading effect is treated phenomenologically with taking a statistical assumption into account. Description of directnucleon-decay properties of the above-mentioned excitations (including giant resonances) is a unique feature of the proposed model, which in applying to closed-shell nuclei is arranged for practical implementations.
\end{abstract}

\section{Introduction}

A great variety of high-energy particle-hole-type excitations, including giant resonances (GRs), is characterized by three main relaxation modes. They are: (i) the particlehole (p-h) strength distribution, or Landau damping, which is a result of the shell structure of nuclei; (ii) coupling of (p-h)-type states to the single-particle (s. p.) continuum that leads to direct nucleon decay and related phenomena; (iii) coupling of (p-h)-type states to many-quasi-particle configurations, or chaotic states, that leads to the spreading effect. Actually, the interplay of these relaxation modes is being changed with increasing the excitation energy. Giant resonances correspond to collective (p-h)-type excitations.

As applied to description of GR damping, we developed a semi-microscopic approach based on the continuumRPA (cRPA) versions of the Migdal's finite Fermi-system theory [1]. Within this approach Landau damping and coupling to the s. p. continuum are described microscopically, using a mean field and $\mathrm{p}-\mathrm{h}$ interaction, while the spreading effect is phenomenologically taken into account directly in the cRPA equations for energy-averaged quantities in terms of the imaginary part of an effective s. p. opticalmodel potential. Such a method allows one to realize the statistical assumption: after energy averaging different (ph)-type states, having the same angular momentum and parity, "decay" into chaotic states independently of one another. In implementations of the approach to description of GR main properties a phenomenological mean field and the Landau-Migdal p-h interaction bound by some selfconsistency conditions are used. The imaginary part of the effective s. p. optical-model potential determines contribution of the spreading effect to the GR main characteristics together with the corresponding real part found from a proper dispersive relationship. The intensity of the imaginary part, which is parameterized as a universal function, exhibiting the saturation-like energy dependence, is adjusted to describe the experimental GR strength distribution. In applying to such a description the semi-microscopic approach is intermediate between "fully microscopic"

\footnotetext{
a e-mail: urin@theor.mephi.ru
}

(chaotic states are substituted by a limited number of $2 \mathrm{p}$ $2 \mathrm{~h}$ configurations) and semi-classical (the shell structure of nuclei is ignored) approaches. The unique feature of the semi-microscopic approach is a possibility to describe direct-nucleon-decay properties of GRs without the use of specific adjustable parameters. Formulation and a diversity of implementations of the semi-microscopic approach, which is valid in the "pole" approximation (i.e., at the close range of the GR energy), are reviewed in [2,3].

To extend the above-described approach on arbitrary (but high enough) excitation energies and also to verify validity of this approach in the energy region of a given GR, we formulate in brief terms a new semi-microscopic model without using additional model parameters. We call this model, as the particle-hole dispersive optical model (PHDOM), in view of a methodical similarity with formulation of the well-known single-quasiparticle dispersive optical model [4]. In particular, the starting point in formulations of both models is the equation for the corresponding manybody Green function (GF). Preliminary considerations of the PHDOM are given in $[3,5]$.

\section{Generalization of the cRPA standard version}

Within the PHDOM the standard and non-standard versions of the cRPA are generalized to take the spreading effect phenomenologically into account. The cRPA standard version is formulated in terms of the local p-h GF $A\left(x, x_{1} ; \omega\right)(x$ is the set of s. p. coordinates, including the spin and isospin variables; $\omega$ is the excitation energy) [6]. The free p-h GF $A_{0}\left(x, x_{1} ; \omega\right)$, which is determined by a mean field (via the s. p. energies $\varepsilon_{\lambda}$, wave functions $\phi_{\lambda}(x)$, GFs $\left.g\left(x, x^{\prime} ; \varepsilon\right)\right)$ and the ground-state s. p. occupation numbers $n_{\lambda}$, and also by a p-h interaction $F\left(x, x_{1}\right)$ are the input quantities for calculating $A\left(x, x_{1} ; \omega\right)$ from the Bethe-Goldstone-type equation. The alternative (and fully equivalent) effective-field method, which is used within the cRPA version of the Migdal's finite Fermi-system theory [1], is actually based on the relationship: $\left[A(\omega) V_{0}\right]=\left[A_{0}(\omega) V(\omega)\right]$. 
Here, $V(x, \omega)$ is the effective field, corresponding to an external s. p. field $V_{0}(x)$ of a given spin-angular and isospin symmetry; the brackets [...] mean integration over one set of s. p. coordinates. The equation for $V$, which follows from the equation for $A$, is widely used [1]. The basic quantities $A$ and/or $V$ determine within the cRPA standard version the integral characteristics of (p-h)-type excitations: the strength distribution and transition density.

To take the above-mentioned statistical assumption into account, we start formulation of the PHDOM with choosing the non-local p-h GF $\mathcal{A}\left(x, x^{\prime} ; x_{1}, x_{1}^{\prime} ; \omega\right)$, as the basic quantity $[5,3]$. In accordance with its spectral expansion $[1,3]$, this GF carries information on (p-h)-type excitations. In particular, it determines the strength function $S_{\mathcal{V}_{0}}(\omega)=-(1 / \pi) \operatorname{Im}\left[. .\left[\mathcal{V}_{0}^{+} \mathcal{A}(\omega) \mathcal{V}_{0}\right] ..\right]$, corresponding to a non-local external field $\mathcal{V}_{0}\left(x, x^{\prime}\right)$ (possibly, $\mathcal{V}_{0}\left(x, x^{\prime}\right)=$ $\left.V_{0}(x) \delta\left(x-x^{\prime}\right)\right)$, and the transition density matrix $\rho\left(x, x^{\prime} ; \omega\right)$. Being considered only in the continuum region, this quantity determines the strength function, as $S_{\mathcal{V}_{0}}(\omega)=$ $\left|\left[\left[\rho(\omega) \mathcal{V}_{0}\right]\right]\right|^{2}$. Accordingly to their spectral expansions, the free GFs $A_{0}$ and $\mathcal{A}_{0}$ are determined by the same input quantities. However, contrary to the free transition densities (appeared in the expansion for $A_{0}$ ), the different transition density matrices $\rho_{\lambda \mu}^{0}\left(x, x^{\prime}\right)=\left(1-n_{\lambda}\right) n_{\mu} \phi_{\lambda}^{*}(x) \phi_{\mu}\left(x^{\prime}\right)$ are orthogonal.

The Bethe-Goldstone-type equation for $\mathcal{A}$

$$
\mathcal{A}(\omega)=\mathcal{A}_{0}(\omega)+\left[. .\left[\mathcal{A l}_{0}(\omega) \mathcal{F}(\omega) \mathcal{A}(\omega)\right] . .\right]
$$

contains the p-h interaction $\mathcal{F}(\omega)=\mathcal{F}_{l-r}+\mathcal{F}_{\text {spr }}(\omega)$. The interaction $\mathcal{F}_{l-r}\left(x, x^{\prime} ; x_{1}, x_{1}^{\prime}\right)=F\left(x, x_{1}\right) \delta\left(x-x_{1}\right) \delta\left(x_{1}-x_{1}^{\prime}\right)$ leads to long-range correlations, including formation of GRs. The interaction $\mathcal{F}_{s p r}(\omega)=\pi(\omega)-\pi(0)$ appears due to coupling of (p-h)-type states to many-quasi-particle configurations. The fragmentation scattering amplitude, or polarization operator, $\pi$ is described by a full set of diagrams irreducible in the $\mathrm{p}$-h channel and, therefore, it takes into account the interference of spreading particles and holes. Because $2 \mathrm{p}-2 \mathrm{~h}$ configurations are the doorway states for spreading of (p-h)-type states, the quantity $\pi\left(x, x^{\prime} ; x_{1}, x_{1}^{\prime} ; \omega\right)$ is a convolution of the $2 \mathrm{p}-2 \mathrm{~h}$ (local) GF $B\left(x_{1}, x_{2}, x_{3}, x_{4} ; \omega\right)$ with a "residual" pair interaction $H^{\prime}\left(x, x_{1}\right): \pi(\omega)=$ [[H'B( $\left.\left.B) H^{\prime}\right]\right]$. The spectral expansion of $\pi(\omega)$ follows from that for the GF $B(\omega)$ and exhibits a high density of poles, corresponding to chaotic states [3]. The p-h interaction $\mathcal{F}_{\text {spr }}(\omega)$ cannot be obviously calculated microscopically. Only the energy-averaged quantity $\overline{\mathcal{F}}_{s p r}(\omega)$, having an imaginary part, can be reasonably parameterized

$$
\begin{aligned}
\overline{\mathcal{F}}_{s p r}\left(x, x^{\prime} ; x_{1}, x_{1}^{\prime} ; \omega\right) & =\left(-i \mathcal{W}\left(x, x^{\prime} ; \omega\right)+\mathcal{P}\left(x, x^{\prime} ; \omega\right)\right) \\
& \times \delta\left(x-x_{1}\right) \delta\left(x^{\prime}-x_{1}^{\prime}\right) .
\end{aligned}
$$

Supposing the same coordinate dependence of $\mathcal{W}$ and $\mathcal{P}$, i.e. $\mathcal{W}\left(x, x^{\prime} ; \omega\right)=W(\omega) f\left(x, x^{\prime}\right)$ and $\mathcal{P}\left(x, x^{\prime} ; \omega\right)=P(\omega)$. $f\left(x, x^{\prime}\right)$, one can get from the spectral expansion of $\pi(\omega)$ a dispersive relationship for $P(\omega)$ as the functional of $W(\omega)$. Within the semi-microscopic approach to description of GR damping an universal function $W(\omega)$, having a saturation-like energy dependence, is used [2,3]. The corresponding expression for $P(\omega)$ is obtained in [7]. To ensure validity of the statistical assumption, the form-factor $f\left(x, x^{\prime}\right)$ should be taken as a constant in the nuclear volume (in implementations of the PHDOM we take $f\left(x, x^{\prime}\right)=f_{\mathrm{W} S}(x)$.
$f_{\mathrm{W} S}\left(x^{\prime}\right)$ or $f\left(x, x^{\prime}\right)=f_{\mathrm{W} S}(x)$, where $f_{\mathrm{W} S}(x)$ is the WoodsSaxon function). Being related to free p-h states $|\lambda \mu\rangle$, the statistical assumption is then realized, as follows:

$$
\begin{aligned}
\left\langle\lambda^{\prime} \mu^{\prime}\left|\overline{\mathcal{F}}_{s p r}(\omega)\right| \lambda \mu\right\rangle & =(-i W(\omega)+P(\omega))\left[\left[\rho_{\lambda^{\prime} \mu^{\prime}}^{0 *} f \rho_{\lambda \mu}^{0}\right]\right] \\
& \simeq(-i W(\omega)+P(\omega)) f_{\lambda} f_{\mu} \delta_{\lambda \lambda^{\prime}} \delta_{\mu \mu^{\prime}},
\end{aligned}
$$

where $f_{\lambda}=\left[\phi_{\lambda}^{*} f_{\mathrm{WS}} \phi_{\lambda}\right]$.

The solution of the energy-averaged basic equation (1) can be sought after the following identical transformation:

$$
\left.\overline{\mathcal{A}}(\omega)=\overline{\mathcal{A}}_{0}(\omega)+\left[. .\left[\overline{\mathcal{A}}_{0}(\omega) \mathcal{F}_{l-r} \overline{\mathcal{A}}(\omega)\right)\right] . .\right],
$$

where the auxiliary quantity $\overline{\mathcal{A}}_{0}(\omega)$ satisfies the equation

$$
\overline{\mathcal{A}}_{0}(\omega)=\mathcal{A}_{0}(\omega)+\left[. .\left[\mathcal{A}_{0}(\omega) \overline{\mathcal{F}}_{s p r} \overline{\mathcal{A}}_{0}(\omega)\right] . .\right] .
$$

In view of (3) this equation, corresponding to the model non-interacting damping quasiparticles, can be solved and the result is given below

$$
\begin{aligned}
& \overline{\mathcal{A}}_{0}\left(x, x^{\prime} ; x_{1}, x_{1}^{\prime} ; \omega\right)=\sum_{\lambda \mu} \bar{A}_{\lambda \mu}(\omega) \phi_{\lambda}(x) \phi_{\mu}^{*}\left(x^{\prime}\right) \phi_{\mu}\left(x_{1}^{\prime}\right) \phi_{\lambda}^{*}\left(x_{1}\right), \\
& \bar{A}_{\lambda \mu}(\omega)=\frac{\left(n_{\lambda}-n_{\mu}\right)}{\left(\varepsilon_{\lambda}-\varepsilon_{\mu}-\omega+\left(n_{\lambda}-n_{\mu}\right)(i W(\omega)-P(\omega)) f_{\lambda} f_{\mu}\right)} .
\end{aligned}
$$

After transition to the local limit in Eqs. (4), (6), i.e., $x^{\prime} \rightarrow$ $x ; x_{1}^{\prime} \rightarrow x_{1}$, we get the equation for the energy-averaged local p-h GF $\bar{A}\left(x, x_{1} ; \omega\right)$

$$
\bar{A}(\omega)=\bar{A}_{0}(\omega)+\left[\left[\bar{A}_{0}(\omega) F \bar{A}(\omega)\right]\right] .
$$

Starting from Eq. (6), one can present the GF $\bar{A}_{0}$ in the form, allowing to take approximately the s. p. continuum into account, using the s. p. optical-model GFs $\bar{g}\left(x, x^{\prime} ; \varepsilon_{\lambda} \pm\right.$ $\omega)$. This rather cumbersome expression for $\bar{A}_{0}$ (explicitly given in [3]) together with Eq. (7) for $\bar{A}$, or with the equivalent equation for the energy-averaged effective field $\bar{V}$ (defined by the relationship $\left.\left[\bar{A}(\omega) V_{0}\right]=\left[\bar{A}_{0}(\omega) \bar{V}(\omega)\right]\right)$ presents generalization of the cRPA standard version on taking the spreading effect into account. In particular, the GF $\bar{A}$ determines the energy-averaged strength functions in the continuum region: $\bar{S}_{V_{0}}(\omega)=\overline{\left|\left[\rho(\omega) V_{0}\right]\right|^{2}}$, where $\overline{\left(\rho^{*}(x, \omega)\right.}$ $\overline{\left.\rho\left(x^{\prime}, \omega\right)\right)}=-(1 / \pi) \operatorname{Im} \bar{A}\left(x, x^{\prime} ; \omega\right)$.

In conclusion of this section we note that the "pole" approximation exploited within the semi-microscopic approach to the description of GR damping corresponds to using the basic quantity $\bar{A}_{\lambda \mu}^{(\text {pole })}(\omega)$, which is following from the second expression of Eq. (6) after substitution $\left(n_{\lambda}-\right.$ $\left.n_{\mu}\right) \rightarrow-1$ in the numerator of this expression. In other words, $\bar{A}_{0}^{(\text {pole })}(\omega)=A_{0}(\omega \rightarrow \omega+i W(\omega)-P(\omega))[2,3]$.

\section{Generalization of the cRPA non-standard version}

Being developed to describe direct-decay properties of various GRs, the cRPA non-standard version was formulated 
within the effective-field method in terms of the amplitudes of "direct + semi-direct" (DSD) reactions induced by an external field $V_{0}(x)$ and related to population of one-hole state $\mu^{-1}$ of the product nucleus: $M_{V_{0}, c}(\omega)=\left[\Psi_{c, 0}^{(+)} V(\omega)\right]$ [2,3]. Here, $V(x, \omega)$ is the corresponding effective field, $\Psi_{c, 0}^{(+)}(x)=n_{\mu} \phi_{\varepsilon_{c}}^{+}(x) \phi_{\mu}(x)$ is the free (local) reaction-channel wave function (w.f.) with $\phi_{\varepsilon_{c}}^{(+)}(x)$ being the nucleon continuum-state w.f. $\left(\varepsilon_{c}=\omega+\varepsilon_{\mu}\right)$, "c" is the set of reactionchannel quantum numbers.

To describe phenomenologically the spreading effect on direct-decay properties of (p-h)-type states at arbitrary energies with taking the statistical assumption into account, we consider the non-local quantities $\psi_{c, 0}^{(+)}\left(x, x^{\prime}\right), \mathcal{V}\left(x, x^{\prime} ; \omega\right)$, $\mathcal{M}_{V_{0}, c}(\omega)=\left[\left[\psi_{c, 0}^{(+)} \mathcal{V}(\omega)\right]\right]=\left[\left[\psi_{c}^{(+)}(\omega) \mathcal{V}_{0}\right]\right]$. Here, the effective (non-local) reaction-channel w.f. $\psi_{c}^{(+)}\left(x, x^{\prime} ; \omega\right)$ satisfies the equation similar to Eq. (1) for $\mathcal{A}(\omega)$, so that the solution for the corresponding energy-averaged quantity can be sought in a similar way

$$
\bar{\psi}_{c}^{(+)}(\omega)=\bar{\psi}_{c, 0}^{(+)}(\omega)+\left[\left[\overline{\mathcal{A}}_{0}(\omega) \mathcal{F}_{l-r} \bar{\psi}_{c}^{(+)}(\omega)\right]\right]
$$

(compare with Eq. (4)), where $\bar{\psi}_{c, 0}^{(+)}\left(x, x^{\prime} ; \omega\right)$ satisfies the auxiliary equation

$$
\bar{\psi}_{c, 0}^{(+)}(\omega)=\psi_{c, 0}^{(+)}+\left[\left[\mathcal{A}_{0}(\omega) \overline{\mathcal{F}}_{s p r}(\omega) \bar{\psi}_{c, 0}^{(+)}(\omega)\right]\right]
$$

(compare with Eq. (5)). The solution of this equation can be sought in the form $\bar{\psi}_{c, 0}^{(+)}\left(x, x^{\prime} ; \omega\right)=n_{\mu} \bar{\phi}_{\varepsilon_{c}}^{(+)}(x) \phi_{\mu}\left(x^{\prime}\right)$ with taking the statistical assumption into account. The result consists in the integral equation for the s. p. OM continuumstate wave function, which is satisfies the equivalent differential equation:

$$
\left\{H_{0}(x)-\left(\varepsilon_{c}+(i W(\omega)-P(\omega)) f_{\mu} f_{W S}(x)\right)\right\} \bar{\phi}_{\varepsilon_{c}}^{(+)}(x)=0 .
$$

Going to the local limit within the expression $\overline{\mathcal{M}}_{\mathcal{V}_{0}, c}=$ $\left[\left[\bar{\psi}_{c}^{(+)}(\omega) \mathcal{V}_{0}\right]\right]$, we get the corresponding expression for the energy-averaged DSD-reaction amplitude $\bar{M}_{V_{0}, c}(\omega)=$ $\left[\bar{\Psi}_{c}^{(+)}(\omega) V_{0}\right]$, which can be further transformed: $\bar{M}_{V_{0}, c}(\omega)=$ $\left[\bar{\Psi}_{c, 0}^{(+)} \bar{V}(\omega)\right]$. Here, the energy-averaged effective field satisfies the equation

$$
\bar{V}(\omega)=V_{0}+\left[\left[F \bar{A}_{0}(\omega) \bar{V}(\omega)\right]\right],
$$

which is first mentioned in Sec. 2.

Apart from the energy-averaged DSD-reaction cross sections, the squared reaction amplitudes determine the partial branching ratios $b_{c}(\delta)$ for partial nucleon decay of (ph)-type states in the channel "c" from an excitation energy interval $\delta$

$$
b_{c}(\delta)=\int_{\delta} \bar{S}_{V_{0}, c}(\omega) d \omega \mid \int_{\delta} \bar{S}_{V_{0}}(\omega) d \omega .
$$

Here, $\bar{S}_{V_{0}, c}(\omega)=\left|\bar{M}_{V_{0}, c}(\omega)\right|^{2}$ is the energy-averaged decaychannel strength function (the fluctuating part of the energyaveraged DSD-reaction cross section is neglected), $\bar{S}_{V_{0}}(\omega)$ is the ordinary strength function discussed in Sec. 2. In view of the spreading effect, the total branching ratio $b_{t o t}(\delta)$ $=\sum_{c} b_{c}(\delta)$ is less than unity. Therefore, the difference $1-$ $b_{t o t}$, which is proportional to $W(\omega)$, can be considered as the branching ratio for statistical (mainly neutron) decay.

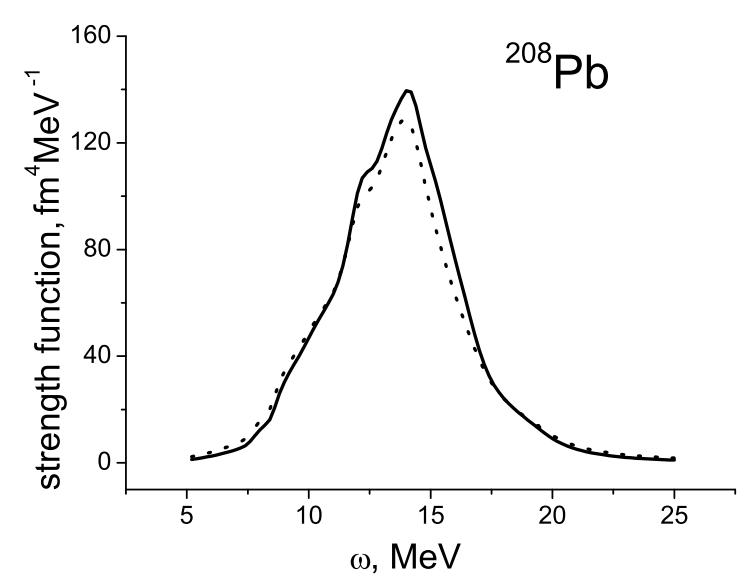

Fig. 1. The isoscalar monopole strength functions calculated for ${ }^{208} \mathrm{~Pb}$

\section{Conclusive remarks}

The equations of the PHDOM for the basic quantities $\bar{A}_{0}\left(x, x^{\prime} ; \omega\right), \bar{\psi}_{c, 0}^{(+)}\left(x, x^{\prime} ; \omega\right)$ and $\bar{A}\left(x, x^{\prime} ; \omega\right), \bar{V}(x, \omega), \bar{M}_{V_{0}, c}(\omega)$ are given above in a rather schematic form. In practice, it is necessary to separate in these equations the spin-angular and isobaric variables. This straightforward procedure used within the semi-microscopic approach to description of GR damping for closed-shell nuclei is described, e. g. in [3]. As a result, one can get the corresponding PHDOM radial equations having as the same structure, as the schematic equations have. First implementations of the PHDOM are given in [8] as applied to neutron radiative capture accompanied by excitation of the isovector giant dipole and quadrupole resonances. Here we show only one example concerned with calculations of the isoscalar monopole strength functions $\bar{S}_{V_{0}}(\omega)$ and $\bar{S}_{V_{0}}^{(\text {pole })}(\omega)\left(V_{0}=r^{2} Y_{00}\right)$ in a vicinity of the isoscalar monopole GR in ${ }^{208} \mathrm{~Pb}$ (see figure 1 , the solid and dotted lines, respectively) ${ }^{1}$. The ratio $R(\omega)=2\left(\bar{S}_{V_{0}}(\omega)-\bar{S}_{V_{0}}^{(\text {pole })}(\omega)\right) /\left(\bar{S}_{V_{0}}(\omega)+\bar{S}_{V_{0}}^{(\text {pole })}(\omega)\right)$ illustrates the difference of the results obtained within the PHDOM and semi-microscopic approach to the description of GR damping (figure 2). As expected, the relative difference $R(\omega)$ is noticeable at the GR "tails" where the considered strength functions are relatively small.

In conclusion, in the present work we formulate in brief terms the new semi-microscopic model (particlehole dispersive optical model) to describe the main relaxation modes of high-energy particle-hole-type excitations in medium-heavy nuclei. Within this model, which is the extension and verification of the previously developed semi-microscopic approach to the description of giant resonance damping, Landau damping and the singleparticle continuum are considered microscopically, while the spreading effect is treated phenomenologically with taking the statistical assumption into account. The model is valid at arbitrary (but high enough) excitation energies. The description of direct-nucleon-decay properties of above-mentioned excitations (including giant resonances) is a unique feature of the proposed model, which in applying to closed-shell nuclei is arranged for practical imple-

\footnotetext{
${ }^{1}$ Details of calculations are briefly given in [8].
} 


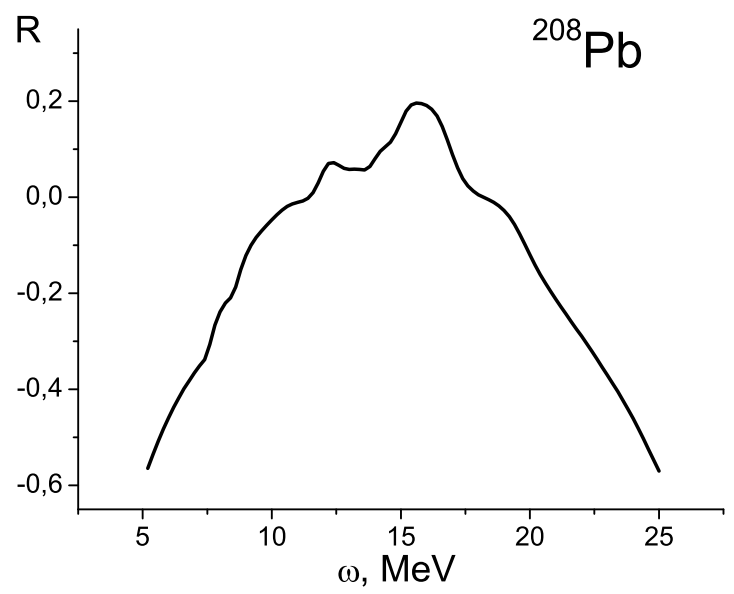

Fig. 2. The relative difference of the isoscalar monopole strength functions calculated for ${ }^{208} \mathrm{~Pb}$ within the PHDOM and semimicroscopic approach to the description of GR damping.

mentations. The detailed description of the model will be published elsewhere.

\section{Acknowledgements}

The author is thankful to I.V. Safonov for his kind help in preparing the manuscript. This work is partially supported by the Russian Foundation for Basic Research under grant no. 12-02-01303-a.

\section{References}

1. A.B. Migdal, Theory of Finite Fermi Systems and Applications to Atomic Nuclei (Nauka, Moscow, 1983, 2nd ed.; Intersci., New York, 1967)

2. M.H. Urin, Nucl. Phys. A 811, 107 (2008)

3. M.G. Urin, Phys. At. Nucl. 74, 1189 (2011)

4. C. Mahaux and S. Sartor, Adv. Nucl. Phys. 20, 1 (1991)

5. M.G. Urin, Phys. At. Nucl. 73, 1384 (2010); M.H. Urin, arXiv: 1005.2349v1 (2010)

6. S. Shlomo and G. Bertsch, Nucl. Phys. A 243, 507 (1975)

7. B.A. Tulupov and M.G. Urin, Phys. At. Nucl. 72, 737 (2009)

8. B.A. Tulupov and M.H. Urin, in the present Proceedings of the Intl. Conference on Nuclear Structure and Related Topics (NSRT12), (Dubna, Russia, July 2-7, 2012) 